\title{
A rare case of neuro-Behçet's disease presenting with limbic encephalitis
}

\author{
Dilcan Kotan, ${ }^{1}$ Sinem Sağ, ${ }^{2}$ Belma Doğan Güngen, ${ }^{3}$ Pınar Polat ${ }^{3}$ \\ ${ }^{1}$ Department of Neurology, Medical Faculty of Sakarya University, Sakarya, Turkey \\ ${ }^{2}$ Department of Rheumatology, Sakarya University Training and Research Hospital, Sakarya, Turkey \\ ${ }^{3}$ Department of Neurology, Sakarya University Training and Research Hospital, Sakarya, Turkey
}

Received: April 2016 Accepted: May 2016

\begin{abstract}
Behçet's syndrome is a recurrent systemic inflammation with an unknown etiology. The onset of the condition often occurs in the second decade of life and it is more common in males than in females. One of the most feared organ involvements associated with BS is neurological involvement, which often affects the brain stem, although hemispheric involvement may be primary in a limited number of patients. Parenchymal neurological involvement in BS affects the prognosis unfavorably, as it may result in severe sequelae or mortality. Early diagnosis and treatment is important to prevent sequelae. Herein, we report the first case of neuro-Behçet's disease presenting with limbic encephalitis who received early stage immunotherapy.
\end{abstract}

Keywords: Intravenous immunglobulin; limbic encephalitis; neuro-Behçet's disease.

Behçet's syndrome (BS) is a condition which often manifests with recurrent oral aphthae, genital ulcerations, and uveitis of joints, vascular and gastrointestinal involvement, and it may also affect the central nervous system (CNS). On the other hand, liver, kidneys, heart, and peripheral nerves are organs which are relatively less affected by BS. ${ }^{[1,2]}$ Neurological involvement occurs after a mean duration of five years following the onset of systemic findings in BS. The condition is three to four times more common in men than in women, and it mainly presents with CNS involvement. Neurological complications may be related to secondary systemic involvement or treatments of BS. Central nervous system involvement in BS comes in the form of parenchymal and non-parenchymal CNS involvement. Cranial magnetic resonance imaging (MRI) is the most common imaging modality used in the evaluation of CNS involvement. ${ }^{[2,3]}$

Limbic encephalitis (LE) was first described by Brierley in 1960 and its association with cancer was first noted in 1968, which was characterized by subacute progressive impairment of short-term memory, psychiatric features, and seizures. ${ }^{[4]}$ Limbic encephalitis is extremely difficult for diagnosis, to be delayed for weeks. The temporal lobe seizures is non-specific. ${ }^{[4,5]}$ The MRI show that temporal lobe abnormalities such as hyperintense signals on $\mathrm{T}_{2}$-weighted or fluidattenuated inversion recovery (FLAIR) sequences, atrophic temporal-limbic structures on $\mathrm{T}_{1}$-weighted images, typically without contrast-enhancement in the brain parenchyma or leptomeninges. ${ }^{[6]}$ Limbic encephalitis is a rare paraneoplastic syndrome which is associated with cancer. The diagnostic test should be the detection of specific onconeural autoantibodies in the cerebrospinal fluid (CSF) and serum. It has been reported that antibody negativity is present in $30 \%$ of cases, and there are antibodies which are unable to be detected in 5 to $10 \%$ of patients. ${ }^{[4,6]}$

Herein, we report the first case of neuro-Behçet's disease (NBD) presenting with LE as the initial manifestation of neurological involvement.

\section{CASE REPORT}

A 36-year-old male patient was admitted to the emergency department presenting with a glassy look, uninterested behavior, communication difficulty,

Corresponding author: Dilcan Kotan, MD. Sakarya Üniversitesi Tip Fakültesi, Nöroloji Anabilim Dalı, 54050 Sakarya, Turkey. 

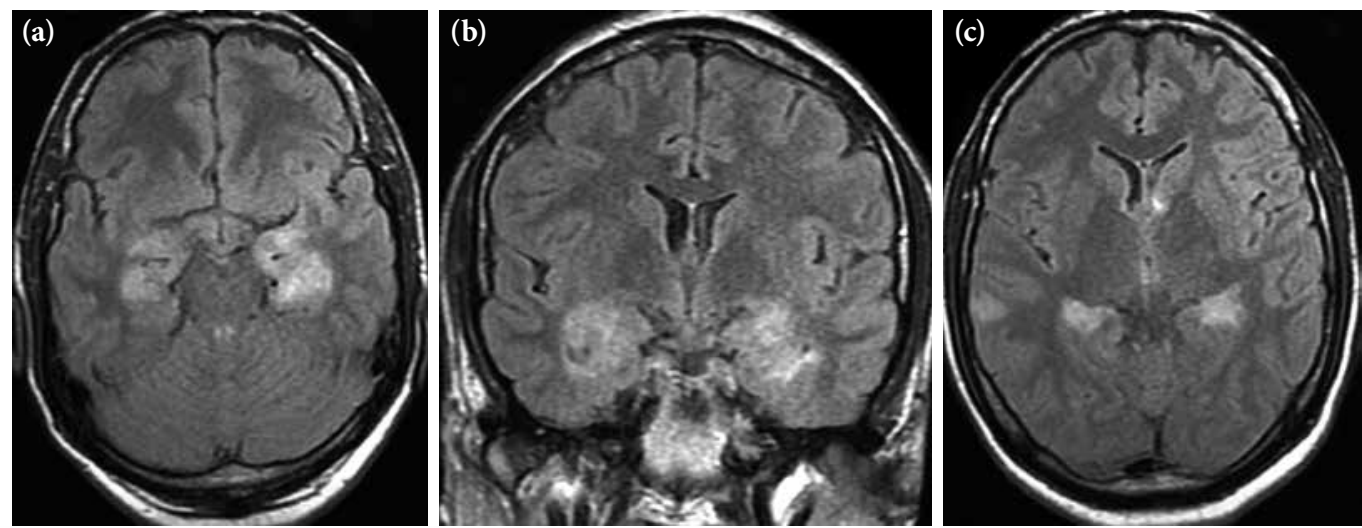

Figure 1. (a-c) Magnetic resonance imaging showing bilateral mesial temporal lobes, amigdala, hippocampus, and the inferior gyrus of the temporal lobe on $\mathrm{T}_{2}$-weighted sections and fluid-attenuated inversion recovery sections showing hyperintense lesion.

and gait disturbance. His medical history revealed the initiation of cyclosporine, colchicine, and methylprednisolone through oral route. The patient was diagnosed with BS based on complaints of recurrent oral sores, joint pain, and blurred vision in both eyes nine years previously. Cyclosporine was discontinued two years ago and treatment was switched to oral azathioprine. The patient reportedly developed uveitis three times during the previous two years and continued using cyclosporine irregularly at his own discretion. His family history included NBD diagnosis in his brother and paternal uncle. His habits included smoking 10 cigarettes per day. Neurological examination revealed cooperation difficulty and uninterested response to questions with the occasional sudden interruptions of conversation. There were no signs of neck stiffness and meningeal irritation. All four extremities were actively moving. The patient was able to walk without support; however, his tandem gait was unable to be assessed due to insufficient cooperation. His plantar reflex was bilaterally indifferent.

Microscopic and biochemical results of the CSF were found to be normal. On cranial MRI, a lesion without contrast uptake was observed in the bilateral mesial temporal lobes, amigdala, hippocampus, and the inferior gyrus of the frontal lobe which was hyperintense on $\mathrm{T}_{2}$-weighted sections and FLAIR sections (Figure 1a-c). Herpes simplex virus (HSV) polymerase chain reaction (PCR) test result was found to be negative for two times. During followup, amnesia was observed. The patient was suspected of developing LE, and NMDA, AMPA1, AMPA2, CASPR2, LGI, GABA-B1, Anti-Hu, Anti-Yo, Anti-Ri, Anti-PNMA2/Ta, Anti-CV2.1, and anti-amphiphysin were found to be negative. All tumor screening tests were also found to be negative. The psychometric tests measuring the functions of orientation, attention, and memory were indicating a severe
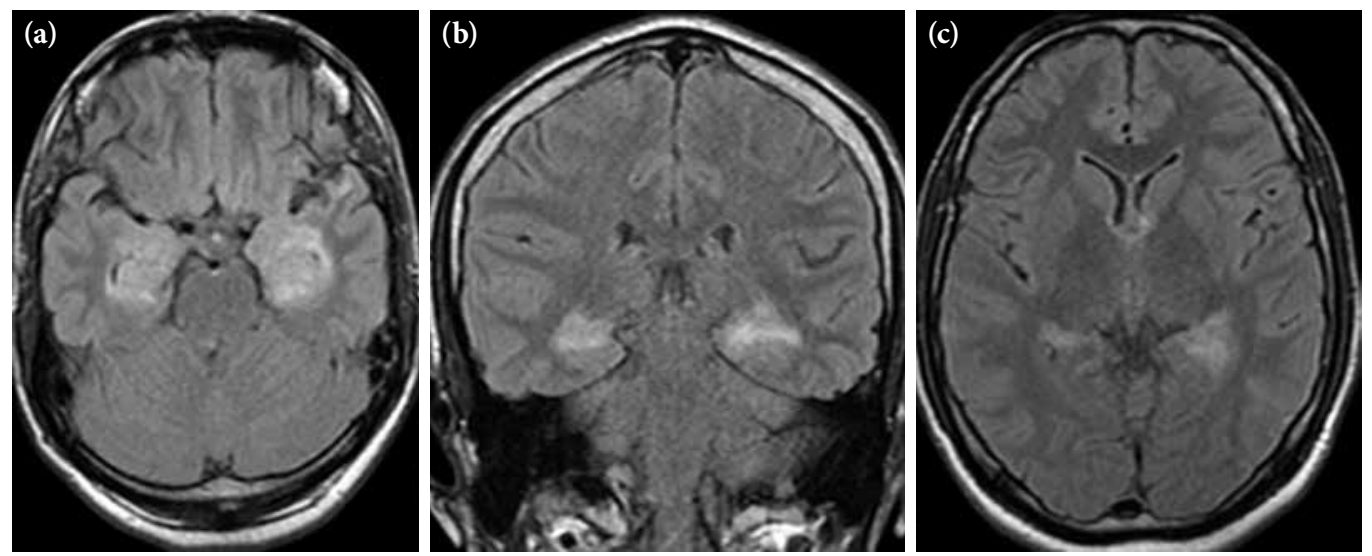

Figure 2. (a-c) Follow-up magnetic resonance imaging showing significant radiological improvement. 
cognitive dysfunction. He received intravenous immunoglobulin (IVIG) $(0.4 \mathrm{~g} / \mathrm{kg}$ daily for five days) and $1 \mathrm{~g}$ of methylprednisolone daily for five days, followed by tapering doses of prednisone over four weeks. Long-term follow-up showed a marked improvement in the clinical picture. In addition, a significant radiological improvement was observed by repeated MRI at four months (Figure 2a-c).

\section{DISCUSSION}

Neuro-Behçet's disease with parenchymal CNS involvement occurs most commonly in the form of brain stem syndrome. ${ }^{[2]}$ Apart from the brain stem, spinal cord involvement may also be seen alone or more commonly with brain stem involvement or cognitive disorder, and the latter exhibits a more severe course. ${ }^{[7,8]}$ In a limited number of cases, hemispheric involvement may be primary, while approximately $10 \%$ of the cases may present with a clinical presentation similar to multiple sclerosis. ${ }^{[9]}$ Indicators of poor prognosis in NBD include parenchymal CNS involvement, increased protein and cells in CSF, extensive lesion involvement, spinal involvement, and progressive course starting from the onset. ${ }^{[10]}$ The case presented herein had parenchymal and extensive lesion involvement. Despite our prediction of poor prognosis, he exhibited a dramatic response to treatment and remained in remission during the follow-up period of nearly nine months, except for a single uveitis episode. Cerebrospinal fluid usually has a high protein content and/or pleocytosis in the cases with parenchymal involvement in NBD. ${ }^{[2]}$ Our case exhibited normal CSF investigation, despite parenchymal involvement.

For the differential diagnosis of NBD, one should bear in mind CNS infections, multiple sclerosis, young cases of stroke and rarely, CNS tumors. Our case presented to hospital with cognitive symptoms, and was diagnosed as clinical and radiological, although serological test results were negative. Our case with LE had approximately the bilateral mesial temporal lobes, amigdala, hippocampus, and other white-matter involvement (Figure 1a-c), whereas the lesions were predominantly periventricular in multiple sclerosis.

Limbic encephalitis is characterized with a lesion in the mesial temporal lobe without contrast uptake on MRI and is manifested with a clinical picture including epileptic seizure, behavioral change, irritability, and memory disorder. The late diagnosis may lead to the patient suffering permanent deficits. The onconeural antigens are undetectable in a subpopulation of symptomatic patients. ${ }^{[1]}$ The most patients with LE are initially diagnosed with herpes simplex encephalitis. Therefore, clinicians must distinguish the two syndromes clinically and serologically. ${ }^{[7,11]}$ Our case was found to be negative for two times with herpes PCR.

In patients in whom LE is considered, tumor screening, despite antibody negativity, is important. ${ }^{[6]}$ Our case had cognitive symptoms. In addition, LE tends to have a better response to immunotherapy. Our case was taken immunotherapy and intravenous (IV) methylprednisolone. The treatment could be delayed, while waiting for paraneoplastic panels, HSV PCR and other diagnostic studies. ${ }^{[12,13]}$ We recommend immunotherapy empirically at the onset of the presentation, as in our case.

Furthermore, LE is associated with frequently neoplasms (e.g. small-cell lung cancer, followed by germ cell tumor of the testis, thymoma, Hodgkin's lymphoma, breast cancer, and teratoma of the ovaries). ${ }^{[14,15]}$ However, we could not find any tumor associated with LE in our case.

Treatment of LE is similar to the treatment of acute episodes in NBD. The treatment of parenchymal CNS involvement usually consists of $1 \mathrm{~g}$ /day IV methylprednisolone for five days, which may be repeated once a week for four weeks. If the patient exhibits indicators of poor prognosis or if a second neurological episode occurs, an immunosuppressant agent should be added to the treatment. Our patient also received IV methylprednisolone in combination with IVIG treatment. However, as the patient experienced an episode on azathioprine, the course of his treatment was switched to monthly $1 \mathrm{~g} /$ day IV cyclophosphamide to control neurological symptoms. The disease remained in remission during the followup period of nearly nine months, except for a single uveitis episode.

In conclusion, this case is the first case of LE associated with NBD in the literature. It is important to recognize the syndrome as the first manifestation of an underlying occurrence of BS.

\section{Declaration of conflicting interests}

The authors declared no conflicts of interest with respect to the authorship and/or publication of this article.

\section{Funding}

The authors received no financial support for the research and/or authorship of this article. 


\section{REFERENCES}

1. Inaba G. Behcet's disease. In: Vinken PJ, Bruyn GW, Klawans H, editors. Handbook of Clinical Neurology. Vol. 12. Amsterdam: Elsevier Science; 1989. p. 593-610.

2. Akman-Demir G, Serdaroglu P, Tasçi B. Clinical patterns of neurological involvement in Behçet's disease: evaluation of 200 patients. The Neuro-Behçet Study Group. Brain 1999;122:2171-82.

3. Akman-Demir G, Bahar S, Coban O, Tasci B, Serdaroglu P. Cranial MRI in Behçet's disease: 134 examinations of 98 patients. Neuroradiology 2003;45:851-9.

4. Corsellis JA, Goldberg GJ, Norton AR. "Limbic encephalitis" and its association with carcinoma. Brain 1968;91:481-96.

5. Brierley JB, Corsellis JAN, Hierons R, Nevin S. Subacute encephalitis of later adult life mainly affecting the limbic areas. Brain 1960;83:357-68.

6. Tüzün E, Dalmau J. Limbic encephalitis and variants: classification, diagnosis and treatment. Neurologist 2007;13:261-71.

7. Gultekin SH, Rosenfeld MR, Voltz R, Eichen J, Posner JB, Dalmau J. Paraneoplastic limbic encephalitis: neurological symptoms, immunological findings and tumour association in 50 patients. Brain 2000;123:1481-94.

8. Siva A, Kantarci OH, Saip S, Altintas A, Hamuryudan V,
Islak C, et al. Behçet's disease: diagnostic and prognostic aspects of neurological involvement. J Neurol 2001;248:95103.

9. Al-Fahad SA, Al-Araji AH. Neuro-Behcet's disease in Iraq: a study of 40 patients. J Neurol Sci 1999;170:105-11.

10. Kural-Seyahi E, Fresko I, Seyahi N, Ozyazgan Y, Mat C, Hamuryudan $\mathrm{V}$, et al. The long-term mortality and morbidity of Behçet syndrome: a 2-decade outcome survey of 387 patients followed at a dedicated center. Medicine (Baltimore) 2003;82:60-76.

11. Vincent A, Buckley C, Schott JM, Baker I, Dewar BK, Detert $\mathrm{N}$, et al. Potassium channel antibody-associated encephalopathy: a potentially immunotherapy-responsive form of limbic encephalitis. Brain 2004;127:701-12.

12. Honnorat J, Antoine JC. Paraneoplastic neurological syndromes. Orphanet J Rare Dis 2007;2:22.

13. Dalakas MC. B cells as therapeutic targets in autoimmune neurological disorders. Nat Clin Pract Neurol 2008;4:557-67.

14. Dalmau J, Rosenfeld MR. Paraneoplastic syndromes of the CNS. Lancet Neurol 2008;7:327-40.

15. Bataller L, Kleopa KA, Wu GF, Rossi JE, Rosenfeld MR, Dalmau J. Autoimmune limbic encephalitis in 39 patients: immunophenotypes and outcomes. J Neurol Neurosurg Psychiatry 2007;78:381-5. 